\title{
Effects of ABCB1 DNA methylation in donors on tacrolimus blood concentrations in recipients following liver transplantation.
}

\author{
Chengcheng $\mathrm{Shi}^{1}$, Liang $\mathrm{Yan}^{1}$, Jie Gao ${ }^{1}$, Shitong $\mathrm{Chen}^{2}$, and Li-Rong Zhang ${ }^{2}$ \\ ${ }^{1}$ Zhengzhou University First Affiliated Hospital \\ ${ }^{2}$ Zhengzhou University
}

November 29, 2021

\begin{abstract}
Aims: To investigate the effects of ABCB1 DNA methylation in donors on individual differences in tacrolimus blood concentrations following liver transplantation. Methods: Twenty-three donor liver samples carrying the CYP $3 \mathrm{~A} 5 * 3 /{ }^{*} 3$ genotype were classified into two groups based on the initial tacrolimus concentration/dose (C0/D) ratio following liver transplantation. ABCB1 mRNA levels in liver tissues and HepG2 cells were determined by qRT-PCR. DNA methylation status in liver tissues and HepG2 cells was determined using Illumina 850 methylation chip sequencing technology and pyrosequencing. 5-Aza-2dC was used to reverse methylation in HepG2 cells. Intracellular tacrolimus concentrations were determined by liquid mass spectrometry. Results: Genome-wide methylation sequencing and pyrosequencing analyses showed that the methylation levels of three ABCB1 CpG sites (cg12501229, cg00634941, and cg05496710) were significantly different between groups with different tacrolimus C0/D ratios. ABCB1 mRNA expression in donor livers was found to be positively correlated with tacrolimus C0/D ratio $(\mathrm{r}=0.458, \mathrm{P}<0.05)$. After treatment with 5-Aza-2-Dc, the methylation levels of the ABCB1 CpG sites in HepG2 cells significantly decreased, and this was confirmed by pyrosequencing; there was also a significant increase in ABCB1 transcription, and this most likely induced a decrease in intracellular tacrolimus concentrations. Conclusions: ABCB1 CpG site methylation affects tacrolimus metabolism in humans by regulating ABCB1 expression. Therefore, ABCB1 DNA methylation in donor livers might be an important epigenetic factor that affects tacrolimus blood concentrations following liver transplantation.
\end{abstract}

\section{INTRODUCTION}

The P-glycoprotein (P-gp) transporter, which is encoded by the $A B C B 1$ transporter gene (also known as the MDR1 gene), is widely distributed in intestinal epithelial cells, liver cells, and renal proximal tubule epithelial cells ${ }^{1}$. Previous studies have shown that $A B C B 1$ may affect tacrolimus absorption, distribution, and excretion ${ }^{2,3}$. Thus, some studies have focused on evaluating the relationship between $A B C B 1$ gene polymorphism (1236C > T, rs1128503, Gly412Gly; 677G > T/A, rs2032582, Ala893DSer/Thr; 3435C > T, rs1045642, Ile1145Ile of exons 12, 21, and 26) and tacrolimus pharmacokinetics ${ }^{3,4}$. However, the findings of these studies are inconsistent as concerns both SNPs and haplotypes, and systematic mechanistic studies to support their conclusions are still lacking. In addition, recent studies carried out on ABCB1 have shown that it functions mainly in prolonging drug retention time in intestinal cells by pumping drugs into the intestinal lumen i.e. on the surface of the gastrointestinal tract, and this also affects tacrolimus absorption ${ }^{5-7}$. However, the effects of $A B C B 1$ on tacrolimus metabolism in liver cells have not been clearly elucidated.

Although $C Y P 450$ and transporter (eg. $A B C B 1$ ) gene polymorphisms are important for individual variations in drug metabolism and pharmacodynamics, they do not fully explain such individual differences ${ }^{8,9}$. In a previous study [in publishing progress], we recruited 78 patients with significantly different initial tacrolimus blood concentrations $\left(\mathrm{C}_{0}>10 \mu \mathrm{g} / \mathrm{L}\right.$ or $\left.<5 \mu \mathrm{g} / \mathrm{L}\right)$ following liver transplantation at the First Affiliated Hospital of Zhengzhou University, and corresponding donor liver samples were collected for the genotyping of CYP3A5 and other genes which have been reported in the literature to be possibly related 
to tacrolimus metabolism(Tables $\mathbf{S} 1$ and $\mathbf{S 2}$ ). We found significant individual differences in tacrolimus plasma concentrations in CYP3A5 non-expressors $\left(C Y P 3 A 5^{*} 3{ }^{*} 3\right)$, indicating that other key factors may also affect tacrolimus metabolism (Figure S1, S2) . In recent times, the influence of epigenetic factors on drug metabolism has received increasing research attention; among these factors, DNA methylation has become a new research hotspot. DNA methylation can alter gene expression through the external regulatory pathway without altering the primary structure of DNA, thereby affecting the metabolism of drugs and endogenous substances ${ }^{10}$. More specifically, cytosine on the $\mathrm{CpG}$ island combines with a methyl group transferred by DNA methyltransferase (DNMT) to produce methyl cytosine, which then inhibits DNA transcription ${ }^{11}$. Previous studies have shown that DNA methylation is an important epigenetic factor that affects CYP450 gene expression ${ }^{12,13}$. In a previous study, we also found DNA methylation to play an important role in $C Y P 3 A 4$ transcriptional regulation ${ }^{14}$. In addition, anti-tumour drugs, such as daunorubicin, activate $A B C B 1$ transcription by hypomethylating its promoter region, and this possibly results in multi-drug resistance ${ }^{15}, 16$.

Therefore, for this study, we selected the liver tissues of 23 donors carrying the $C Y P 3 A 5^{*} 3 /{ }^{*} 3$ genotype and exhibiting varied initial tacrolimus blood concentrations. DNA methylation sequencing was performed to screen for the different methylation sites of drug metabolism enzymes or drug transporters (such as $A B C B 1$ ), and then we evaluated the relationship between the different methylation sites and the tacrolimus $\mathrm{C}_{0} / \mathrm{D}$ ratio. In addition, by treating HepG2 cells with methylase inhibitors, we further verified whether DNA methylation is a key epigenetic factor that affects tacrolimus metabolism.

\section{MATERIALS AND METHODS}

Patients and Sample collection

All the liver transplantation recipients included in this study met the following criteria: age $>18$ years, postoperative survival time [?] 3 months, normal liver function within 1 month following surgery, and patients having received treatment with tacrolimus in combination with steroids and mycophenolate mofelil after transplantation. The corresponding donors met the following criteria: hepatitis or other infectious disease free, normal liver and kidney function, and no treatment with any drug known to interact with tacrolimus within the two previous weeks.

Tacrolimus was administered orally at a dose of $0.05 \mathrm{mg} / \mathrm{kg} / \mathrm{d}$ twice daily from the first day following transplantation. Routine therapeutic drug monitoring (TDM) for tacrolimus was initiated $48 \mathrm{~h}$ after the administration of its first dose. The initial tacrolimus concentration $\left(\mathrm{C}_{0}\right)$ in whole blood was determined through an enzyme amplification immunoassay using the EMIT 2000 tacrolimus assay kit (Siemens Healthcare Diagnostics, DE, USA) on the day of blood collection. The tacrolimus $\mathrm{C}_{0} / \mathrm{D}$ ratio was calculated by dividing the tacrolimus concentration by the corresponding weight-adjusted dose. Liver samples of the included donors were collected during liver trimming at the time of transplantation, cut into small pieces, and immediately stored in liquid nitrogen.

DNA methylation microarray screening

The DNAs of 15 donor livers carrying the $C Y P 3 A 5^{*} 3$ / $^{*} 3$ genotype were extracted (approximately $500 \mathrm{ng}$ of DNA per sample) and sent to Shanghai Jingzhou Genomics Technology Co., Ltd., where genome-wide DNA methylation was assessed using the Illumina Infinium Human Methylation850K BeadChip (Illumina Inc., USA) according to the manufacturer's instructions. Array data (.IDAT files) were analysed using the ChAMP function in $\mathrm{R}$ for the determination of methylation levels. The methylation status of all probes was denoted as the $\beta$ value, which is the ratio of the methylated probe intensity to the overall probe intensity (the sum of the methylated and unmethylated probe intensities plus the constant, $\alpha$, where $\alpha=100$ ). CpG sites with $|\Delta \beta|$ [?] 0.20 (in test vs. control) and adjusted $P$ value [?] 0.05 , were considered to be differentially methylated sites. A CpG site was considered to be hypermethylated if its $\Delta \beta$ was [?]0.20, or hypomethylated if its $\Delta \beta$ was [?] - 0.20 . The average $\beta$ values for promoters and $\mathrm{CpG}$ islands were compared between Group1 (G1: low tacrolimus $\mathrm{C}_{0} / \mathrm{D}$ ratio group) and Group2 (G2: high tacrolimus $\mathrm{C}_{0} / \mathrm{D}$ ratio group). Promoters and CpG islands with $|\Delta \beta|[?] 0.20$ and adjusted $P$ value [?] 0.05 , were retained for further analysis. 


\section{Pyrosequencing analysis}

Genomic DNA (500 ng) extracted from the 23 CYP3A $5^{*} 3{ }^{*} 3$ genotype donor livers was transformed by sodium bisulfite using the EZ DNA Methylation Kit (Zymo Research, Orange County, California, USA) and further purified using the Wizard DNA Clean-up System (Promega, Madison, Wis, USA). The sequences of the primers used for the $A B C B 1$ DNA methylation analysis are shown in Table 1 . Each PCR reaction system consisted of $100 \mathrm{ng}$ of DNA converted by sodium bisulfite, $100 \mathrm{pM}$ deoxyriboside triphosphate, 10 pM positive/reverse primers, and 1 unit of Taq polymerase (Merck KGaA, Darmstadt, Germany), which resulted in a final volume of $25 \mu \mathrm{L}$. After initial denaturation at $95{ }^{\circ} \mathrm{C}$ for $5 \mathrm{~min}$, amplification was performed for 40 cycles at $95{ }^{\circ} \mathrm{C}$ for $30 \mathrm{~s}, 60{ }^{\circ} \mathrm{C}$ for $30 \mathrm{~s}$, and $72{ }^{\circ} \mathrm{C}$ for $30 \mathrm{~s}$, followed by a final elongation step at $72{ }^{\circ} \mathrm{C}$ for 5 min. PCR products were analysed by non-denatured $6 \%$ polyacrylamide gel electrophoresis, following staining with ethidium bromide.

In addition, genomic DNA was extracted from cells treated with the methylation inhibitor, 5-Aza-2-DC, and changes in $A B C B 1$ DNA methylation were also evaluated by pyrosequencing.

Cell culture and treatments

The human hepatocellular carcinoma cell line, HepG2, which was purchased from the China Centre for Type Culture Collection (Wuhan, China), and confirmed by short tandem repeat analysis, was cultured in DMEM (HyClone/Thermo Fisher Scientific, Beijing, China) supplemented with 10\% FBS (LONSA SCIENCE S.R.L., Montevideo, Uruguay). To determine the optimum concentration and administration period for 5-Aza-2-DC (Selleck, Shanghai, China) and tacrolimus (Selleck, Shanghai, China), HepG2 cells were seeded into 12-well plates at a density of $4 \times 10^{4}$ cells/well and cultured for $24 \mathrm{~h}$ at $37^{\circ} \mathrm{C}$ in a $5 \% \mathrm{CO}_{2}$ atmosphere. For treatment with 5-Aza-2-DC, the cells were exposed to 5-Aza-2-DC dissolved in DMSO $(0.1 \% \mathrm{v} / \mathrm{v})$ at a series of final concentrations $(0,0.1,0.5,1.0,2.5,5.0,10$, and $50 \mu \mathrm{M})$ for 24,48 , and $72 \mathrm{~h}$. Then, cell viability was evaluated using the CCK-8 kit (Dojindo, Shanghai, China) according to the manufacturer's instructions, and $A B C B 1$ mRNA and protein expression levels were evaluated by RT-qPCR and Western blotting, respectively. For treatment with tacrolimus, the cells were exposed to tacrolimus dissolved in DMSO $(0.1 \% \mathrm{v} / \mathrm{v})$ at a series of final concentrations $(0,0.01,0.1,1.0,10,50,100$, and $200 \mu \mathrm{M})$ for 24,48 , and $72 \mathrm{~h}$, and cell viability was evaluated using the CCK- 8 kit. Based on the results of the optimum time determined by CCK- 8 analysis, HepG2 cells were treated with $0,40,50,60,70,80,90$, and $100 \mu \mathrm{M}$ tacrolimus for $24 \mathrm{~h}$, and cell viability was evaluated using the CCK- 8 kit to determine the tacrolimus optimum concentration.

\section{5-Aza-2-DC treatment}

HepG2 cells at the logarithmic growth stage $\left(1.2 \times 10^{6}\right.$ cells/well $)$ were spread on 6 -well plates. After $24 \mathrm{~h}$ of culturing, the cells were treated with $0 \mu \mathrm{M}$ (DMSO; $0.1 \% \mathrm{v} / \mathrm{v}$ ) or $10 \mu \mathrm{M}$ 5-Aza-2-DC, and the 5-Aza-2DC-containing medium was replaced every $24 \mathrm{~h}$. After treatment with 5-Aza-2-DC for $72 \mathrm{~h}$, the medium was discarded and replaced with a tacrolimus-containing medium $(60 \mu \mathrm{M})$, and the cells were cultured for another $48 \mathrm{~h}$. After $48 \mathrm{~h}$, the medium was replaced with tacrolimus-free DMEM and the cells were further cultured. Samples of the medium were taken at $0,4,6$, and $12 \mathrm{~h}$ following its replacement. Next, the cells were digested with trypsin for $2 \mathrm{~min}$, and then digestion was terminated by adding more medium. The cells were centrifuged at $300 \times g$ for 3 min and washed thrice with PBS. The supernatant of cell culture and cells were stored at $-80 \operatorname{degC}$ for further evaluation.

Total RNA extraction and RT-qPCR analysis

Total RNA was extracted using the TRIzol Reagent (CoWin Biosciences, Jiangsu, China) according to the manufacturer's instructions. The extracted RNA was reverse-transcribed into cDNA using Super Script III Reverse Transcriptase (Vazyme Biotech Co., Ltd., Nanjing, China). ABCB1 mRNA expression was evaluated through a relative quantitative method. The formula, 2

ABCB1-F: 5'-TTGCTGCTTACATTCAGGTTTCA-3',

ABCB1-R: 5'-AGCCTATCTCCTGTCGCATTA-3', 


\section{GAPDH-F: 5'-GCACCGTCAAGGCTGAGAAC-3',}

\section{GAPDH-R: 5'-TGGTGAAGACGCCAGTGGA-3'.}

Western blot analysis

Lysates of HepG2 cells (which were treated with 5-Aza-2-DC and tacrolimus) were run on $8 \%$ sodium dodecyl sulphate-polyacrylamide gels, transferred to polyvinylidene difluoride membranes, and incubated with P glycoprotein (Abcam Inc. Cambridge, MA). Antibody binding was detected using enhanced chemiluminescence ECL Plus western blotting detection reagents (Merck Millipore Ltd. Tullagreen, Carrigtwohill, Co. Cork, IRELAND).

Determination of tacrolimus intracellular concentrations by liquid-mass spectrometry

The collected cells were resuspended in $100 \mu \mathrm{L}$ of PBS or medium and mixed with $50 \mu \mathrm{L}$ of internal standard (ascomycin, $800 \mathrm{ng} / \mathrm{mL}$, dissolved in methanol), $50 \mu \mathrm{L}$ of methanol, and $500 \mu \mathrm{L}$ of methyl-tert-butyl ether. After vortexing and centrifugation $\left(14000 \times g, 5 \mathrm{~min}, 4^{\circ} \mathrm{C}\right), 450 \mu \mathrm{L}$ of the organic layer was dried on a nitrogen blow-dry apparatus. The extract was redissolved in $50 \mu \mathrm{L}$ of a complex solution $(2 \mathrm{mM}$ ammonium acetate and $0.1 \%$ formic acid). After vortexing and centrifugation $\left(12000 \times g, 10 \mathrm{~min}, 4{ }^{\circ} \mathrm{C}\right)$, the supernatant $(45$ $\mu \mathrm{L}$ ) was placed in a liquid injection flask. The mobile phase for UHPLC-MS/MS detection was [acetonitrile (containing $0.1 \%$ formic acid): $2 \mathrm{mmol} / \mathrm{L}$ ammonium acetate (containing $0.1 \%$ formic acid) $=9: 1$, and the column temperature was $55{ }^{\circ} \mathrm{C}$. The specific product ions were $\mathrm{m} / \mathrm{z} 821.5$ and $\mathrm{m} / \mathrm{z} 809.5$ for tacrolimus and the internal standard, respectively. Ionisation was carried out in positive ion mode with a capillary voltage of $3.5 \mathrm{kV}$, a cone voltage of $22 / 29$ (tacrolimus/internal standard), an ion source temperature of $120{ }^{\circ} \mathrm{C}$, a desolvation temperature of $350{ }^{\circ} \mathrm{C}$, a nitrogen flow rate of $600 \mathrm{~L} / \mathrm{h}$, a collision pressure of $5 \times 10^{-3}$ bar, and a collision energy of $17 / 21$ (tacrolimus/internal standard).

Statistical analysis

The SPSS software (Armonk, New York corporation, USA) was used for data analysis, and GraphPad Prism (version 6.0, San Diego, California, USA) was used to plot graphs. All data are expressed as the mean \pm SD. The independent sample t-test was used to compare and analyse data between the two groups, with homogenous variance of the bivariate normal distribution. One-way analysis of variance was used to analyse the differences between the two groups. Normally distributed bivariate data were analysed using the Pearson correlation test, while non-normally distributed bivariate data were analysed using the Spearman correlation test. $P$ values $<0.05$ were considered statistically significant.

\section{RESULTS}

Whole genome methylation microarray sequencing of CYP3A $5 * 3 / * 3$ genotype donor livers

Through principal component analysis, approximately 800,000 methylation sites were observed in the twodimensional diagram after probe filtering and normalisation (Figure 1A ). Based on the methylation $\beta$ value between the $5 \mathrm{~kb}$ upstream and downstream transcription start sites (TSS), significant differences in methylation levels were observed between groups G1 and G2 (Figure 1B ). In addition, heat and scatter maps showed that there was a large number of methylation sites, with different levels of methylation, between groups G1 and G2 (Figure 1C and D ).

Methylation levels and mRNA expression of ABCB1 in relation to tacrolimus blood concentration

Through methylation chip screening, the methylation levels of three ABCB1 sites (cg12501229, cg00634941, and cg05496710) located on chromosome 7 were found to be significantly different between the high and low tacrolimus blood concentration groups (Figure 2A ). Using the pyrophosphorylation assay, the methylation levels of these three sites were further evaluated in the 23 donor liver specimens carrying the CYP3A5*3/*3 genotype. We found that the methylation levels of these sites in the high $\mathrm{C}_{0} / \mathrm{D}$ group were all significantly lower than those in the low $\mathrm{C}_{0} / \mathrm{D}$ group (Figure 2B-D ). 
Furthermore, $A B C B 1$ mRNA expression levels were determined by RT-qPCR, and correlation analyses revealed that $A B C B 1$ mRNA expression in donor livers was positively correlated with the tacrolimus $\mathrm{C}_{0} / \mathrm{D}$ ratio $(\mathrm{r}=0.458, P<0.05)$ (Figure 3A ). An $A B C B 1 \mathrm{mRNA}$ expression level of 0.15 was used as the cutoff value to analyse the tacrolimus $\mathrm{C}_{0} / \mathrm{D}$ ratio and differences between the low-and high-expression groups (Figure 3B and C ). Our results showed that the greater proportion of patients with high blood tacrolimus concentrations were found in the high $A B C B 1$ expression group.

Effects of 5-Aza-2-DC on ABCB1 expression in HepG2 cells

First, the optimal concentration and administration time for 5-Aza-2-DC were determined (Figure 4A-C ). Then, based on findings in literature, $10 \mu \mathrm{M} 5$-Aza-2-DC was used to investigate the effects of $A B C B 1$ methylation status on its expression in HepG2 cells. We found that after treatment with $10 \mu \mathrm{M} 5$-Aza-2-DC for 24, 48 and $72 \mathrm{~h}, A B C B 1 \mathrm{mRNA}$ and protein expression levels significantly increased at $72 \mathrm{~h}$ (Figure $4 \mathrm{D}-\mathrm{F}$ ). Therefore, $10 \mu \mathrm{M}$ 5-Aza-2-DC and $72 \mathrm{~h}$ were selected as the optimal concentration and treatment duration, respectively, for the determination of the effects of 5-Aza-2-DC on the methylation levels of the three ABCB1 CpG sites (cg12501229, cg00634941, and cg05496710).

Effects of 5-Aza-2-DC on ABCB1 methylation levels and tacrolimus metabolism in HepG2 cells

A pyrophosphorylation assay was performed to investigate the effects of 5-Aza-2-DC on $A B C B 1$ methylation. As shown in Figure 5, we found that the methylation levels of the $A B C B 1$ sites in the 5-Aza-2-DC-treated group $(10 \mu \mathrm{M})$ were significantly lower $(P<0.001)$ than those in the untreated group $(0 \mu \mathrm{M})$. To determine the effects of 5-Aza-2-DC on tacrolimus metabolism, intracellular tacrolimus concentrations were determined by UHPLC-MS/MS. We found the tacrolimus contents of 5-Aza-2-DC-treated cells to be significantly lower than those of untreated cells at 0,4 , and $6 \mathrm{~h}$ following the removal of tacrolimus-containing medium $(P<$ $0.05)$. At $12 \mathrm{~h}$, although there was a slight increase in intracellular tacrolimus concentrations, its levels in the 5-Aza-2-DC-treated group were still significantly lower than those in the untreated group $(0 \mu \mathrm{M})$, and this may be due to its intracellular metabolism in a dynamic equilibrium scenario (Figure 6 ).

\section{DISCUSSION}

Several studies have reported that individual differences in drug response cannot be fully explained by polymorphisms in genes encoding drug-metabolizing enzymes or transporters ${ }^{17}{ }^{18}$. Recently, epigenetic modifications, which regulate the expression of several enzymes and transporters involved in drug metabolism, have been recognised as important factors that affect individual differences in clinical drug response ${ }^{19}$. DNA methylation affects the expression of CYP450 (CYP1A1 , CYP1A2 , CYP1B1 ,CYP2C19 , CYP2D6 , $C Y P 2 E 1$, and $C Y P 2 W 1$ ), thus leading to significant individual differences in enzyme expression ${ }^{20-23}$. In addition, DNA methylation regulates the expression of $A B C G 2$ and $A B C B 1$, which play a crucial role in determining the success or failure of cancer chemotherapy by mediating multi-drug resistance and individual differences in drug transport ${ }^{24,25}$. As most studies on the methylation of genes encoding drug transporters have been carried out in the field of oncology, we investigated, for the first time, whether $A B C B 1$ DNA methylation in donor livers affects tacrolimus plasma concentrations in liver transplant recipients by regulating its expression.

In this study, we analysed 15 donor liver samples carrying the $C Y P 3 A 5^{*} 3 /^{*} 3$ genotype using DNA methylation microarray technology, and we found $A B C B 1$ methylation levels to be correlated with tacrolimus serum concentrations in liver transplantation patients. Based on the findings of previous studies carried out on $A B C B 1$ methylation and tacrolimus metabolism, ${ }^{2,26,} 27$ we speculated that $A B C B 1$ DNA methylation might be another key factor that affects tacrolimus metabolism by regulating $A B C B 1$ expression.

Previous studies have demonstrated that there exists no correlation between the frequency of $A B C B 1$ gene polymorphisms and tacrolimus plasma concentrations following renal transplantation, $, 428,29$ and this is consistent with the findings of one of our previous studies [in publishing progress] . However, our studies found that there exist significant individual differences in tacrolimus plasma concentrations in liver transplant recipients who receive donor livers with the $C Y P 3 A 5^{*} 3 /{ }^{*} 3$ genotype; thus, for the first time, we evaluated the 
methylation status of 23 liver samples carrying the $C Y P 3 A 5^{*} 3 /{ }^{*} 3$ genotype using a methylation microarray assay validated by pyrosequencing. Our findings showed that DNA methylation levels at three $A B C B 1 \mathrm{CpG}$ sites (cg12501229, cg00634941, and cg05496710) in donor livers were significantly different between the high and low tacrolimus $\mathrm{C}_{0} / \mathrm{D}$ ratio groups following liver transplantation. In addition, in consonance with the findings of previous studies, ${ }^{30-32} A B C B 1 \mathrm{mRNA}$ levels in donor livers were found to be negatively correlated with its methylation levels.

To the best of our knowledge, no studies have been carried out on the effects of $A B C B 1$ methylation, especially of its three CpG sites (cg12501229, cg00634941, and cg05496710), on tacrolimus metabolism. Therefore, in this study, the effects of $A B C B 1$ methylation on tacrolimus metabolism were first evaluated using the methylation inhibitor, 5-Aza-2-DC. It was shown that in HepG2 cells, $A B C B 1$ methylation levels at its three methylation sites significantly decreased following treatment with 5-Aza-2-DC. Moreover, tacrolimus intracellular concentrations significantly decreased following treatment with 5-Aza-2-DC. In addition, $A B C B 1 \mathrm{mRNA}$ and protein levels significantly increased following treatment with 5-Aza-2-DC. For clinical samples, the methylation levels of the cg12501229, cg00634941, and cg05496710 sites in the high $\mathrm{C}_{0} / \mathrm{D}$ group were all significantly lower than those in the low $\mathrm{C}_{0} / \mathrm{D}$ group, and $A B C B 1 \mathrm{mRNA}$ expression was found to be positively correlated with tacrolimus $\mathrm{C}_{0} / \mathrm{D}$ ratio. These findings indicate that a decrease in DNA methylation could result in an increase in $A B C B 1$ expression in donor livers, which would lead to an increase in tacrolimus excretion from liver cells and a consequent increase in tacrolimus plasma concentrations of the recipient.

This study had one limitation, namely, we did not construct a methylation-specific expression plasmid to determine which of the three $A B C B 1$ methylation sites plays a leading role in the regulation of gene expression; however, further studies will be conducted on this in the future.

\section{CONCLUSION}

Our study demonstrated that DNA methylation of ABCB1 CpG sites (cg12501229, cg00634941, and cg05496710) could regulate its expression in donor livers, thus inducing individual differences in initial tacrolimus concentrations following liver transplantation.

\section{ACKNOWLEDGEMENT}

We would like to thank Key Laboratory of Hepatobiliary and Pancreatic Surgery and Digestive Organ Transplantation of Henan Province for donor liver samples and data collection. And we would like to thank Wiley Editing Services (https://editingservices.wiley.cn/) for English language editing. This work was supported by the grants from the National Natural Science Foundation of China $(81773815 ; 82073931)$ and the special fund for Health Commission of Henan Province (SB201902017).

\section{COMPETING INTERESTS}

There are no competing interests to declare.

\section{DATA AVAILABILITY STATEMENT}

The data that support the findings of this study are available from the corresponding author upon reasonable request.

\section{ETHICS STATEMENT}

This study was approved by the Ethics Committee of the First Affiliated Hospital of Zhengzhou University (2019-KY-019). Written informed consent was obtained according to the Declaration of Helsinki.

\section{CONTRIBUTIONS}

CCS: conception, design, data collection, analysis of data, drafting, and revision of manuscript. LY: conception, interpretation of results and critical revision of manuscript. STC: analysis of data, interpretation of results. LRZ: design and revision of manuscript. 


\section{REFERENCES}

1. Wu X, Yin C, Ma J, et al. Polyoxypregnanes as safe, potent, and specific ABCB1-inhibitory pro-drugs to overcome multidrug resistance in cancer chemotherapy in vitro and in vivo. Acta Pharm Sin B 2021;11:18851902.

2. Mallina H, Elumalai R, S FDP, et al. Computational validation of ABCB1 gene polymorphism and its effect on tacrolimus dose concentration/levels in renal transplant individuals of South India. Comput Biol Med 2021;139:104971.

3. Azam F, Khan M, Khaliq T, et al. Influence of ABCB1 gene polymorphism on concentration to dose ratio and adverse effects of tacrolimus in Pakistani liver transplant recipients. Pak J Med Sci 2021;37:689-694.

4. Sallustio BC, Noll BD, Hu R, et al. Tacrolimus dose, blood concentrations and acute nephrotoxicity, but not CYP3A5/ABCB1 genetics, are associated with allograft tacrolimus concentrations in renal transplant recipients. Br J Clin Pharmacol 2021;87:3901-3909.

5. Qin XL, Chen X, Zhong GP, et al. Effect of Tacrolimus on the pharmacokinetics of bioactive lignans of Wuzhi tablet (Schisandra sphenanthera extract) and the potential roles of CYP3A and P-gp. Phytomedicine 2014;21:766-72.

6. Li X, Zhao K, Ma N, et al. Association of ABCB1 promoter methylation with aspirin exposure, platelet function, and clinical outcomes in Chinese intracranial artery stenosis patients. Eur J Clin Pharmacol 2017;73:1261-1269.

7. Correa S, Binato R, Du Rocher B, et al. ABCB1 regulation through LRPPRC is influenced by the methylation status of the GC -100 box in its promoter. Epigenetics 2014;9:1172-83.

8. Chen Z, Cheng X, Zhang L, et al. The impact of IL-10 and CYP3A5 gene polymorphisms on doseadjusted trough blood tacrolimus concentrations in early post-renal transplant recipients. Pharmacol Rep 2021;73:1418-1426.

9. Peng W, Lin Y, Zhang H, et al. Effect of ABCB1 3435C > T Genetic Polymorphism on Pharmacokinetic Variables of Tacrolimus in Adult Renal Transplant Recipients: A Systematic Review and Meta-analysis. Clin Ther 2020;42:2049-2065.

10. Kronfol MM, Jahr FM, Dozmorov MG, et al. DNA methylation and histone acetylation changes to cytochrome P450 2E1 regulation in normal aging and impact on rates of drug metabolism in the liver. Geroscience 2020;42:819-832.

11. Chequin A, Costa LE, de Campos FF, et al. Antitumoral activity of liraglutide, a new DNMT inhibitor in breast cancer cells in vitro and in vivo. Chem Biol Interact 2021;349:109641.

12. He H, Wang P, Li SG, et al. [Effects of pargyline on histone methylation in promoter and enhancer regions and transcription of CYP3A4/3A7]. Yao Xue Xue Bao 2017;52:91-8.

13. Lkhagvadorj K, Zeng Z, Meyer KF, et al. Postnatal Smoke Exposure Further Increases the Hepatic Nicotine Metabolism in Prenatally Smoke Exposed Male Offspring and Is Linked with Aberrant Cyp2a5 Methylation. Int J Mol Sci 2020;22.

14. Wang X, Wei L, Yang J, et al. DNA methylation determines the regulation of pregnane X receptor on CYP3A4 expression. Clin Exp Pharmacol Physiol 2021;48:250-259.

15. Onda K, Suzuki R, Tanaka S, et al. Decitabine, a DNA methyltransferase inhibitor, reduces Pglycoprotein mRNA and protein expressions and increases drug sensitivity in drug-resistant MOLT4 and Jurkat cell lines. Anticancer Res 2012;32:4439-44.

16. Li S, Zhang Y, Ho SH, et al. Combination of tumour-infarction therapy and chemotherapy via the co-delivery of doxorubicin and thrombin encapsulated in tumour-targeted nanoparticles. Nat Biomed Eng 
2020;4:732-742.

17. Liu H, Yang ZK, Li Y, et al. ABCB1 variants confer susceptibility to primary open-angle glaucoma and predict individual differences to latanoprost treatment. Biomed Pharmacother 2016;80:115-120.

18. Jonaitis P, Jonaitis L, Kupcinskas J. Role of Genetic Polymorphisms of Cytochrome P450 2C19 in Pantoprazole Metabolism and Pantoprazole-based Helicobacter pylori Eradication Regimens. Curr Drug Metab 2020;21:830-837.

19. Habano W, Kawamura K, Iizuka N, et al. Analysis of DNA methylation landscape reveals the roles of DNA methylation in the regulation of drug metabolizing enzymes. Clin Epigenetics 2015;7:105.

20. Habano W, Gamo T, Sugai T, et al. CYP1B1, but not CYP1A1, is downregulated by promoter methylation in colorectal cancers. Int J Oncol 2009;34:1085-91.

21. Nakajima M, Iwanari M, Yokoi T. Effects of histone deacetylation and DNA methylation on the constitutive and TCDD-inducible expressions of the human CYP1 family in MCF-7 and HeLa cells. Toxicol Lett 2003;144:247-56.

22. Ye L, Xu Y, Wang L, et al. Downregulation of CYP2E1 is associated with poor prognosis and tumor progression of gliomas. Cancer Med 2021.

23. Luo $\mathrm{R}$, Ge $\mathrm{C}$, Xiao $\mathrm{X}$, et al. Identification of genetic variations associated with drug resistance in non-small cell lung cancer patients undergoing systemic treatment. Brief Bioinform 2021;22.

24. Ohmura H, Ito M, Uchino K, et al. Methylation of drug resistance-related genes in chemotherapysensitive Epstein-Barr virus-associated gastric cancer. FEBS Open Bio 2020;10:147-157.

25. Xu L, Wang Y. Combined influence of ABCB1 genetic polymorphism and DNA methylation on aspirin resistance in Chinese ischemic stroke patients. Acta Neurol Belg 2021.

26. Wang J, Huang L, Gao P, et al. Diltiazem on tacrolimus exposure and dose sparing in Chinese pediatric primary nephrotic syndrome: impact of CYP3A4, CYP3A5, ABCB1, and SLCO1B3 polymorphisms. Eur J Clin Pharmacol 2021;77:71-77.

27. Vaclavikova R, Klajic J, Brynychova V, et al. Development of highresolution melting analysis for ABCB1 promoter methylation: Clinical consequences in breast and ovarian carcinoma. Oncol Rep 2019;42:763-774.

28. Wu P, Ni X, Wang M, et al. Polymorphisms in CYP3A5*3 and MDR1, and haplotype modulate response to plasma levels of tacrolimus in Chinese renal transplant patients. Ann Transplant 2011;16:54-60.

29. Yildirim E, Sahin G, Kaltus Z, et al. Effect of CYP3A5 and ABCB1 Gene Polymorphisms on Tacrolimus Blood Concentration in Renal Transplant Recipients. Clin Lab 2019;65.

30. Wu LX, Zhao HB, Wen CJ, et al. Combined Influence of Genetic Polymorphism and DNA Methylation on ABCB1 Expression and Function in Healthy Chinese Males. Eur J Drug Metab Pharmacokinet 2017;42:627634.

31. Wu LX, Wen CJ, Li Y, et al. Interindividual epigenetic variation in ABCB1 promoter and its relationship with ABCB1 expression and function in healthy Chinese subjects. Br J Clin Pharmacol 2015;80:1109-21.

32. Yang J, Zhou JS, Zhao YX, et al. ABCB1 hypomethylation is associated with decreased antiplatelet effects of clopidogrel in Chinese ischemic stroke patients. Pharmazie 2015;70:97-102.

FIGURES AND TABLES

Table 1 Primers used for $A B C B 1$ DNA methylation analysis

\begin{tabular}{llll}
\hline Detected site & Segment & Primer sequence (5'-3') & Product size (bp) \\
cg05496710 & PCR sense & AAGTAGAATATTTAGGGGAGGTATG & 84
\end{tabular}




\begin{tabular}{llll} 
& PCR antisense & ACCTTACATTCTCCAATTCTTTAAAA & \\
Sequencing & GGAGGTATGGGTTTT & \\
$\operatorname{cg} 00634941$ & PCR sense & TGGATTGTTGGATTTGTAGTTTTAATAG & 102 \\
& PCR antisense & AAACTCCAATCCCCTTTACTAATA & \\
$\operatorname{cg} 12501229$ & Sequencing & GTTTTTAGGTGATGTTGAT & \\
& PCR sense & GTGAATAGTTGGTGATAATTATTTATTGTG & 99 \\
& Sequencing & AAATAAACATCTCCTTTTTAAACATTTT & \\
& & TGGTGATAATTATTTATTGTGG & \\
\hline
\end{tabular}

Figure 1 Whole genome methylation microarray sequencing of $C Y P 3 A 5^{*} 3 /{ }^{*} 3$ genotype donor liver. (A) Principal Component Analysis (PCA) diagram; (B) Distribution of methylation degree between the $5 \mathrm{~kb}$ upstream and downstream transcription start sites (TSS); (C) Heat map of differential methylation sites; (D) Scatter plot of different methylation sites. G1: low tacrolimus $\mathrm{C}_{0} / \mathrm{D}$ ratio group; $\mathrm{G} 2$ : high tacrolimus $\mathrm{C}_{0} / \mathrm{D}$ ratio group; $\mathrm{n}=15$.

Figure 2 The methylation sites (red vertical bars) within the $A B C B 1$ methylation region located on chromosome 7 (A) and the methylation level at cg12501229 site (B), cg00634941 site(C), and cg05496710 site (D) of $A B C B 1$ gene in donor livers carried with $C Y P 3 A 5^{*} 3 /{ }^{*} 3$ genotype and different tacrolimus $\mathrm{C}_{0} / \mathrm{D}$ ratio (High: $\mathrm{n}=12$, Low: $\mathrm{n}=11$ ) ; ${ }^{* *} P<0.01$.

Figure 3 Correlation of $A B C B 1$ mRNA expression with tacrolimus $\mathrm{C}_{0} / \mathrm{D}$ ratio in $C Y P 3 A 5^{*} 3 /^{*} 3$ donor livers $(\mathrm{n}=23)$. (A) correlation analyses; $(\mathbf{B}, \mathbf{C})$ An $A B C B 1 \mathrm{mRNA}$ expression level of 0.15 was used as the cut-off value to analyse the tacrolimus $\mathrm{C}_{0} / \mathrm{D}$ ratio $(\mathbf{B})$ and the tacrolimus $\mathrm{C}_{0} / \mathrm{D}$ ratio distribution $(\mathbf{C}) ;{ }^{*} P$ $<0.05$.

Figure 4 Effects of 5-Aza-2-DC on cell viability, $A B C B 1$ expression in HepG2 cells. (A-C) The effects of 5-Aza-2-DC on cell viability after treated at indicated concentrations of 5-Aza-2-DC for $24 \mathrm{~h}(\mathbf{A}), 48 \mathrm{~h}(\mathbf{B}$ ), and 72h (C), respectively; (D-F ) $A B C B 1$ mRNA expression (D) and protein expression (E , F ) after treated with 5-Aza-2-DC $(10 \mu \mathrm{M})$ for $24 \mathrm{~h}, 48 \mathrm{~h}, 72 \mathrm{~h}$ treatment, respectively; ${ }^{*} P<0.05,{ }^{* *} P<0.01,{ }^{* * *} P$ $<0.001$ vs $0 \mu \mathrm{M} 5$-Aza-2-DC (DMSO; $0.1 \% \mathrm{v} / \mathrm{v}$ ).

Figure 5 Effects of 5-Aza-2-DC $(10 \mu \mathrm{M})$ on DNA methylation at three CpG sites located on $A B C B 1$ promoter region in HepG2 cells; ${ }^{* * *} P<0.001$ vs $0 \mu \mathrm{M}$ 5-Aza-2-DC (DMSO; 0.1\% v/v).

Figure 6 Changes of tacrolimus concentration in HepG2 cells over time replaced with tacrolimus-free DMEM after treated with 5 -Aza-2-DC $(0 \mu \mathrm{M}$ or $10 \mu \mathrm{M}) 72 \mathrm{~h}$ and then incubated with $60 \mu \mathrm{M}$ tacrolimus for $48 \mathrm{~h} ;{ }^{*} P$ $<0.05,{ }^{* * *} P<0.001$ vs $0 \mu \mathrm{M}$ 5-Aza-2-DC (DMSO; 0.1\% v/v). 
A

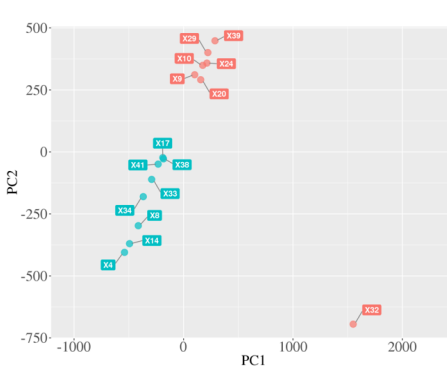

C

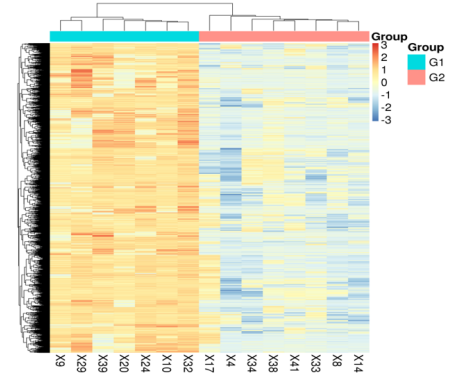

B

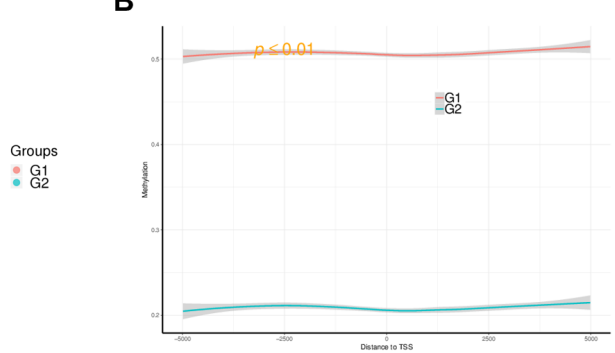

D

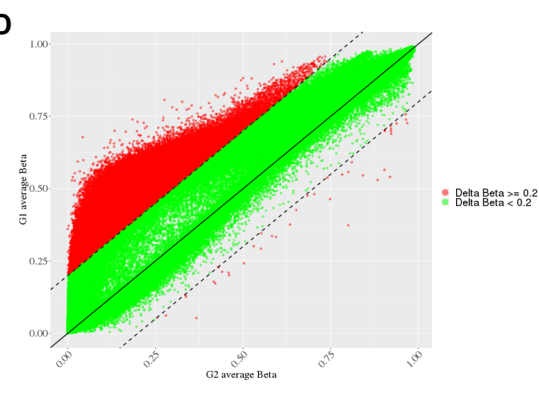

A

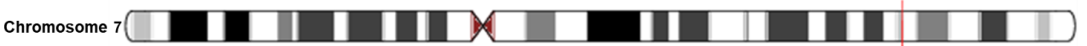

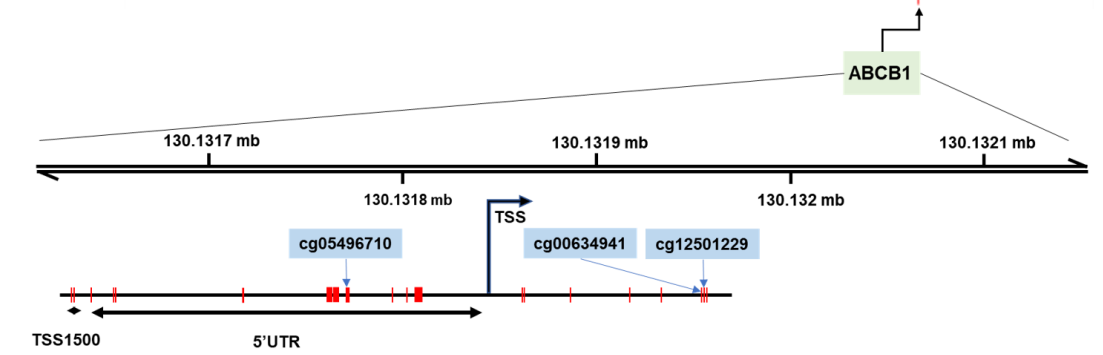

B

C

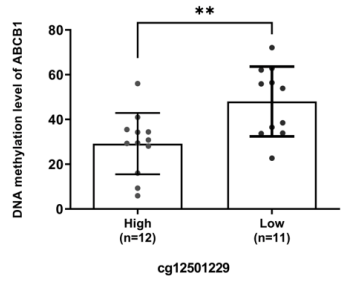

A

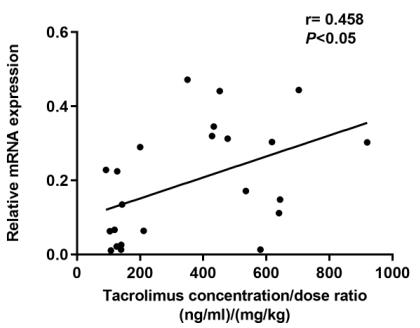

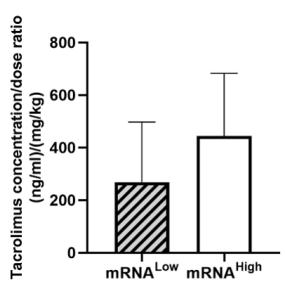

D

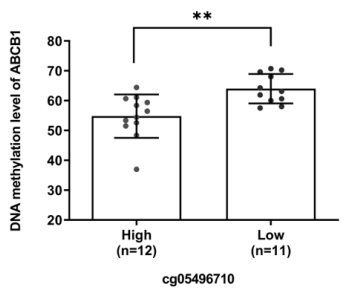

C

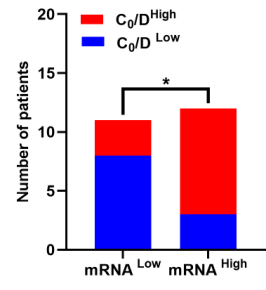


A

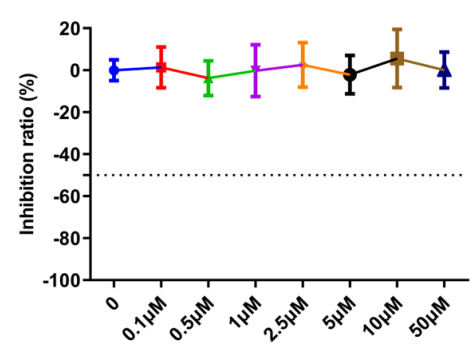

B

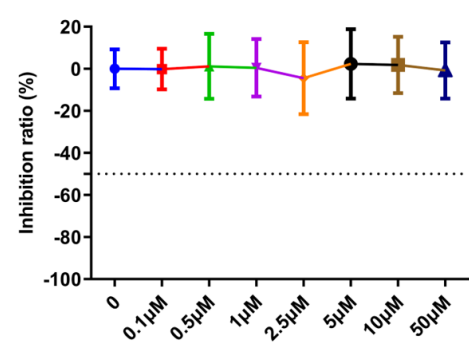

C

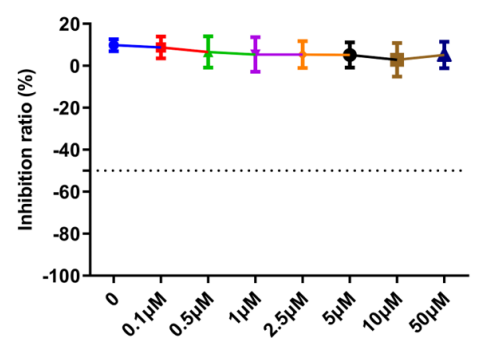

D

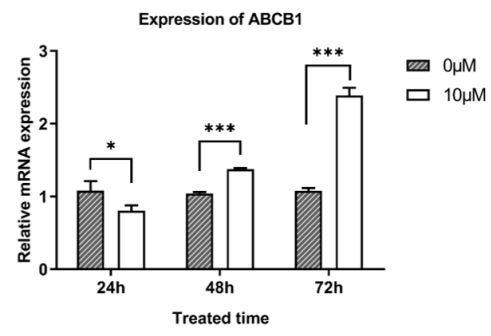

E

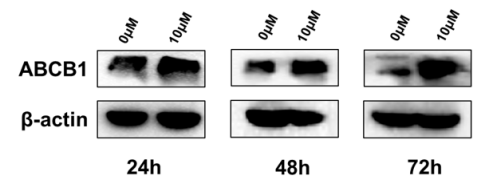

F

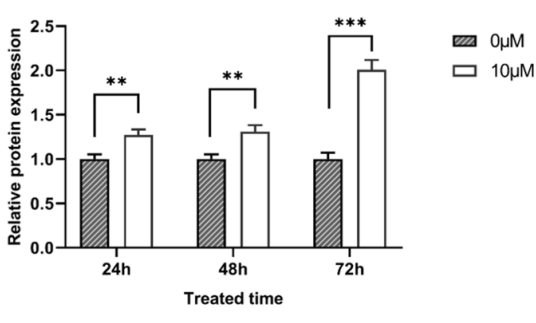

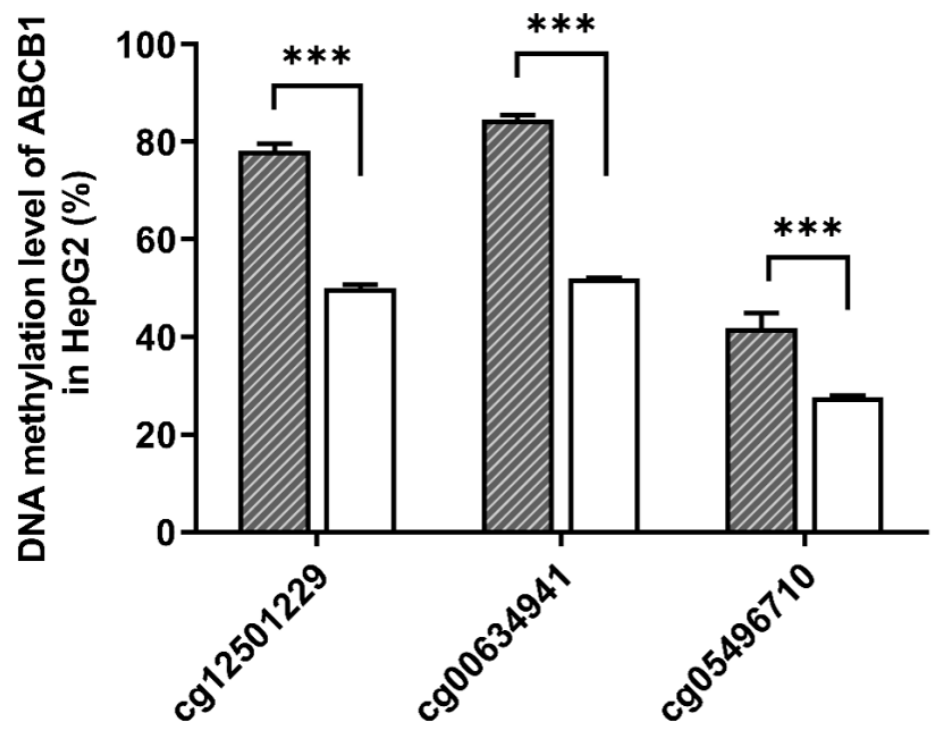




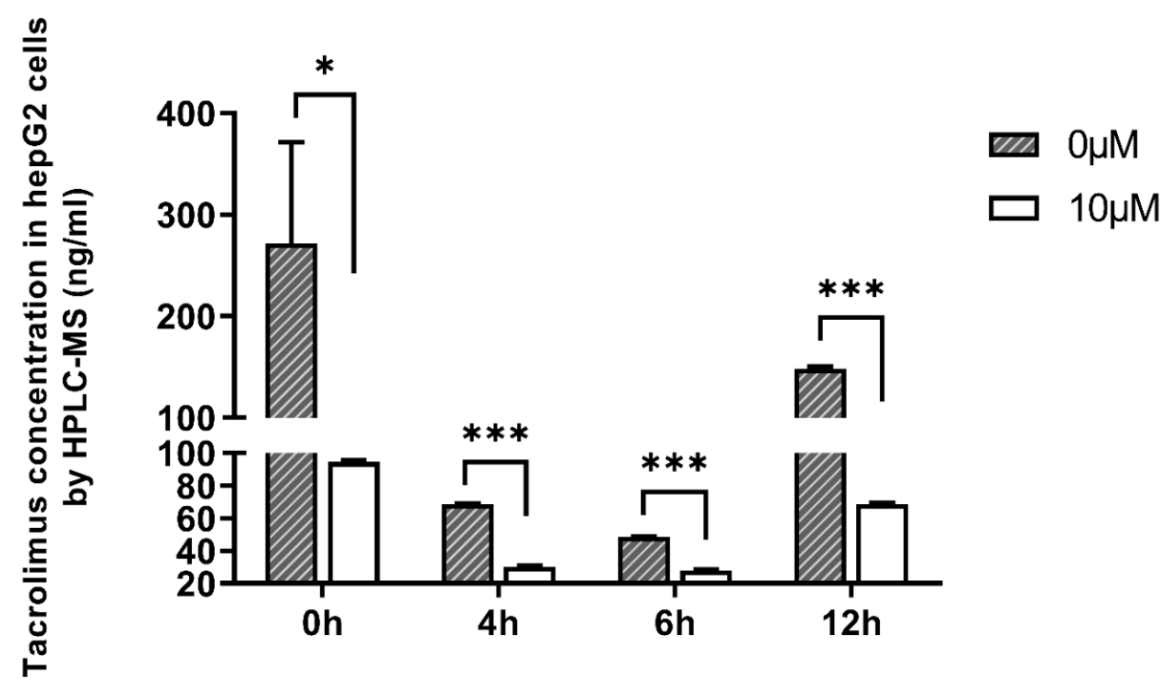

